Zeolites and Related Materials: Trends, Targets and Challenges Proceedings of $4^{\text {th }}$ International FEZA Conference

A. Gedeon, P. Massiani and F. Babonneau (Editors)

(C) 2008 Elsevier B.V. All rights reserved.

\title{
Dynamics of PEO-PPO-PEO block copolymer aggregation and silicate mesophase formation monitored by time-resolved ATR-FTIR spectroscopy
}

\author{
María Teresa Sánchez, Joaquín Pérez-Pariente, Carlos Márquez-Alvarez \\ Instituto de Catálisis y Petroleoquímica (ICP), CSIC, Marie Curie, 2, Cantoblanco, \\ Madrid - 28049, Spain
}

\begin{abstract}
The use of the attenuated total reflectance (ATR) technique to perform time-resolved FTIR spectroscopy analysis of the synthesis of SBA-15 silicate is reported. This spectroscopy is a powerful technique to investigate the assembly of surfactants and silicate species as well as surfactant micellization, as infrared bands are sensitive to local polarity and conformation of the surfactant chains. Critical micellization temperature (CMT), a key parameter that determines mesophase synthesis conditions and long-range ordering, is determined by in situ time-resolved ATR-FTIR under the conditions used to synthesize the mesophase. This technique allows also to determine the dynamics of the hydrolysis and condensation reactions of the silicate precursor.
\end{abstract}

Keywords: SBA-15 synthesis, in situ FTIR, block copolymer surfactant, CMT.

\section{Introduction}

Detailed information about the mechanism of the soft-templating synthesis of ordered mesoporous silicates is required for tailoring the porosity of these materials. In situ techniques, such as small-angle X-ray scattering and diffraction and time-resolved ${ }^{1} \mathrm{H}$ NMR, have been applied to investigate the kinetics and reaction mechanisms of the silicate-surfactant mesophase formation [1,2]. Information complementary to the structural characterization provided by these techniques can be obtained by infrared spectroscopy. The use of the attenuated total reflectance (ATR) technique allows performing time-resolved FTIR spectroscopy characterization of the synthesis gels to determine the dynamics of the hydrolysis and condensation reactions of precursors [3]. Furthermore, this spectroscopy is a powerful technique to investigate the assembly of surfactants and silicate species as well as surfactant micellization, as infrared bands are sensitive to local polarity and conformation of the surfactant chains. Su et al. [4,5] reported transmission FTIR spectra of poly(ethylene oxide)-poly(propylene oxide)poly(ethylene oxide) (PEO-PPO-PEO) block copolymers in aqueous solution recorded at temperatures below and above critical micellization temperature (CMT). Changes in intensities, shapes and location of FTIR absorption bands of the surfactants were related to the progressive dehydration of PPO fragments due to micellization. Compared to transmission cells, time-resolved ATR-FTIR provides a more versatile and simple method to measure CMT, a key parameter that determines mesophase synthesis conditions and long-range ordering [2]. In ATR spectroscopy [6], the infrared beam undergoes total internal reflection through a crystal in contact with the sample. The evanescent wave established beyond the interface penetrates into the sample a short distance, from tenths to several microns, which depends on the diffraction indexes of the 
internal reflection element (IRE) and the sample. Thus, ATR spectroscopy provides a short and highly reproducible pathway that allows performing FTIR analysis even in the presence of solvents that strongly absorb IR. Among the IRE materials commercially available, diamond combines the hardness, chemical and thermal resistance required to withstand the conditions of typical molecular sieve synthesis media.

In this contribution, time-resolved ATR-FTIR spectroscopy is used to investigate the synthesis of the SBA-15 silicate material templated by the PEO-PPO-PEO triblock copolymer Pluronic PE10400 in nitric acid solution, at $\mathrm{pH}$ close to 0 , and to determine the critical micellization temperature (CMT) of PE10400 at the conditions used to synthesize the mesoporous material.

\section{Experimental}

The synthesis of SBA-15 silicate was carried out in a PTFE-lined stainless steel stirred reaction vessel adapted to a horizontal attenuated total reflectance (ATR) accessory (SensIR Technologies DurasamplIR), with a 9-reflection, $4 \mathrm{~mm}$ diameter diamondfaced ZnSe prism. An aqueous solution containing $2.1 \mathrm{wt} \%$ of Pluronic PE10400 (BASF) was prepared by dissolving the surfactant in a $1.3 \mathrm{M} \mathrm{HNO}_{3}$ solution at $55^{\circ} \mathrm{C}$ with stirring for $1 \mathrm{~h}$. The solution was poured into the ATR cell and cooled down to $30^{\circ} \mathrm{C}$ by means of a circulation thermostat. Then, tetraethyl orthosilicate (TEOS, 99\%, Merck) was added to the surfactant solution while stirring to obtain a synthesis gel with molar composition $1 \mathrm{SiO}_{2}: 0.017 \mathrm{PE} 10400: 26 \mathrm{HNO}_{3}: 128 \mathrm{H}_{2} \mathrm{O}$. The temperature was raised to $55^{\circ} \mathrm{C}$ at a mean rate of $1^{\circ} \mathrm{C} / \mathrm{min}$, then kept at this temperature for several hours. Time-resolved in situ ATR-FTIR spectra of the reaction mixture were recorded in the $4000-900 \mathrm{~cm}^{-1}$ wavenumber range, with a resolution of $4 \mathrm{~cm}^{-1}$, using a Thermo Nicolet Nexus FTIR spectrometer equipped with a liquid nitrogen-cooled MCT detector. The low-angle XRD pattern of the solid product obtained after ageing at $55^{\circ} \mathrm{C} / 24 \mathrm{~h}$ and $80^{\circ} \mathrm{C} / 4 \mathrm{~h}$ showed the characteristic peaks of the $2 \mathrm{D}$ hexagonal structure (space group p6 $\mathrm{mm}$ ). Prior to the silicate synthesis, the critical micellization temperature (CMT) of the PE10400 acidic solution was determined. A solution of surfactant in $1.3 \mathrm{M} \mathrm{HNO}_{3}$ was prepared in the same way as for the silicate synthesis, transferred to the ATR cell and cooled down to $4^{\circ} \mathrm{C}$. The solution was then heated to $55^{\circ} \mathrm{C}$ at a constant rate of $1^{\circ} \mathrm{C} / \mathrm{min}$ and time-resolved in situ ATR-FTIR spectra were recorded.

\section{Results and discussion}

In order to obtain the actual CMT value for the surfactant solution that was used to synthesize the mesoporous silicate, time-resolved ATR-FTIR spectra of the $1.3 \mathrm{M}$ $\mathrm{HNO}_{3}$ aqueous solution containing $2.1 \mathrm{wt} \%$ PE10400 (pH $\sim 0$ ) were recorded while heating the solution from 4 to $55^{\circ} \mathrm{C}$. Nitrate ions in solution gave rise to strong absorption bands in the wavenumber range $1320-1420 \mathrm{~cm}^{-1}$, which obscured the $\mathrm{C}-\mathrm{H}$ deformation bands due to the surfactant. Therefore, determination of CMT was based on the analysis of the copolymer C-C and C-O stretching bands. In Figure 1 (left) the series of spectra collected along time have been plotted in the wavenumber range corresponding to the later vibration bands. The most intense band in this region, assigned to C-O-C stretching modes shows a shift toward higher wavenumbers as the temperature increases (Figure 1, right). At low temperatures, the band location is hardly modified. However, the transfer PPO chains from the aqueous medium to the hydrophobic environment of the micellar core when micellization occurs results in a marked shift at temperatures above CMT [4,5]. Taking the onset of this step as the CMT value, a temperature of $21.7^{\circ} \mathrm{C}$ is obtained (Figure 1). This figure is slightly higher than 

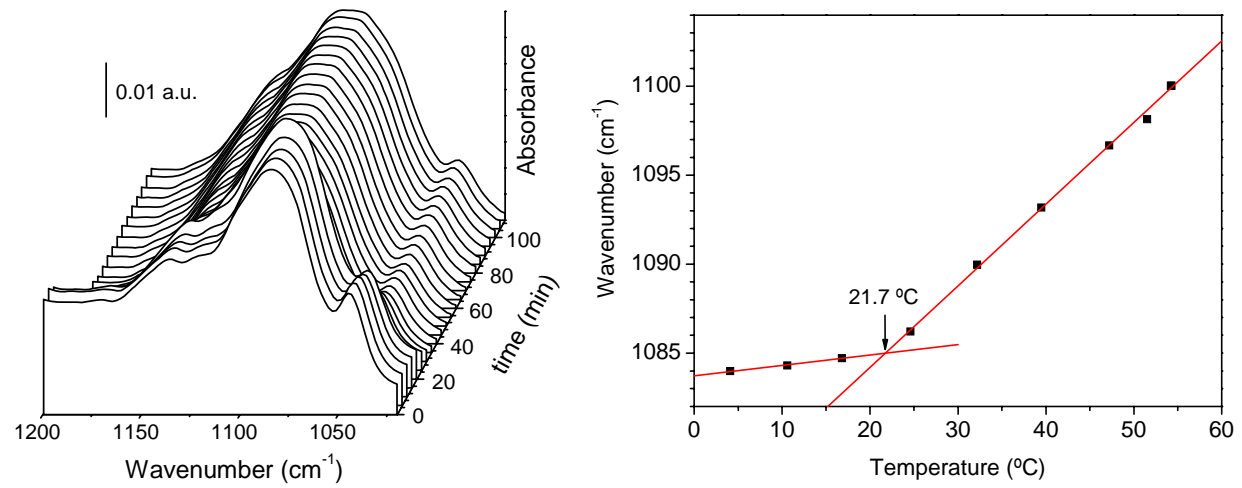

Figure 1. Left: In situ time-resolved ATR-FTIR spectra of an aqueous solution of Pluronic PE10400 (2.1 wt \%) in $1.3 \mathrm{M} \mathrm{HNO}_{3}$ recorded during heating from 4 to $55^{\circ} \mathrm{C}$. Right: Location of the C-O-C stretching band as a function of temperature.

the CMT value determined for a $2.1 \mathrm{wt} \%$ aqueous solution of Pluronic P104 using a dye solubilization method (ca. $20^{\circ} \mathrm{C}$ ) [7]. It can be concluded that micellization is hardly affected by $\mathrm{pH}$.

Figure 2 shows the time-resolved ATR-FTIR spectra recorded during the synthesis of SBA-15. TEOS was added to the cell containing the acidic solution of PE10400 at $30^{\circ} \mathrm{C}$ and spectra were collected along time while temperature was raised to ca. $55^{\circ} \mathrm{C}$. The spectrum collected at zero time corresponds to the surfactant acidic solution at $30^{\circ} \mathrm{C}$. In the wavenumber range from 800 to $1800 \mathrm{~cm}^{-1}$, the spectrum is dominated by the strong absorption bands due to water bending $\left(1636 \mathrm{~cm}^{-1}\right)$ and nitrate stretching modes (doublet at 1388 and $1342 \mathrm{~cm}^{-1}$ ). Weak bands corresponding to the surfactant vibration modes appear superimposed to the nitrate stretching bands and in the $1100-1150 \mathrm{~cm}^{-1}$ range. Following addition of TEOS, bands at 1086, 1044 and $880 \mathrm{~cm}^{-1}$ appear, which can be assigned to ethanol produced by the hydrolysis of TEOS. This reaction seems to be completed in a few minutes, as the intensity of ethanol bands increases quickly and reaches its maximum value in ca. $5 \mathrm{~min}$, when temperature is close to $43^{\circ} \mathrm{C}$ (Figure 2, right). A simultaneous decrease of intensity of the water and nitrate IR bands can be observed, due to dilution as a result of ethanol production. In these spectra, a weak band at $950 \mathrm{~cm}^{-1}$ is observed, which correspond to bending of $\mathrm{Si}-\mathrm{OH}$ groups produced by hydrolysis of the ethoxy species. Also weak bands around $1050-1150 \mathrm{~cm}^{-1}$ are formed, in the Si-O-Si stretching range, indicative of the formation of silicate species due to condensation reactions. The intensity of water, ethanol and nitrate bands remain constant for $20 \mathrm{~min}$ and a subsequent fast decay takes place for several minutes, and a nearly constant level is reached in around $2 \mathrm{~h}$. This decay can be attributed to the precipitation of the silicate-surfactant mesophase. The precipitation would increase the local concentration of the solid silicate-surfactant mesophase in the thin layer in contact with the ATR crystal that interacts with the evanescent wave, and thus shorten the effective pathlength through the aqueous solution. This is confirmed by the marked increase of the surfactant vibration bands that occurs as water and ethanol bands decrease. According to the reaction sequence proposed by Flodström et al. [8], the FTIR data suggest that the surfactant micelles, on which the silicate species formed by the fast hydrolysis and condensation of TEOS in the first few minutes of reaction are adsorbed, would remain associated into flocs in solution for several minutes before precipitation 

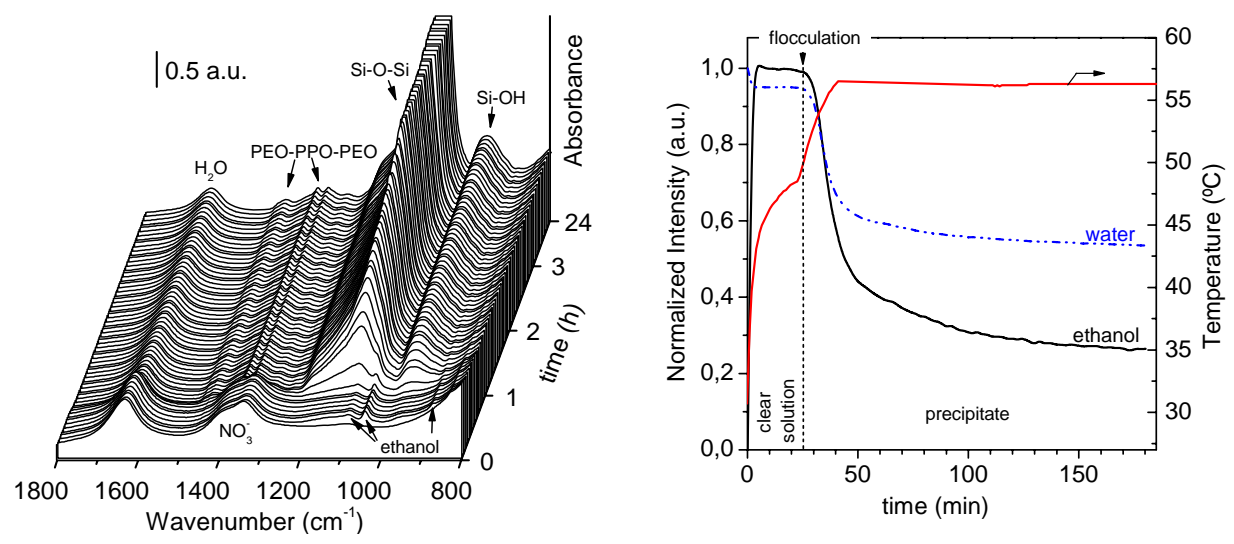

Figure 2. Left: In situ time-resolved ATR-FTIR spectra of the SBA-15 synthesis in a 1.3 M HNO3 aqueous solution containing 2.1 wt $\%$ PE10400. At time $=0$, TEOS was added to the acidic surfactant solution and temperature increased from 30 to $55^{\circ} \mathrm{C}$. Right: Normalized integrated absorbance of IR bands at $1635 \mathrm{~cm}^{-1}$ (water) and $880 \mathrm{~cm}^{-1}$ (ethanol) and temperature of the synthesis mixture versus time.

eventually takes place. After precipitation, the intensity of surfactant vibration bands in the $1300-1450 \mathrm{~cm}^{-1}$ range quickly reach a constant level, while the silicate $\mathrm{Si}-\mathrm{O}-\mathrm{Si}$ stretching and $\mathrm{Si}-\mathrm{OH}$ bending bands show a continuous growth. Furthermore, the most intense silicate stretching band continuously shifts from ca. 1085 to $1066 \mathrm{~cm}^{-1}$, and its relative intensity compared to the $\mathrm{Si}-\mathrm{OH}$ bending band increases. These results evidence the progressive condensation and densification of silicate fragments in the silicatesurfactant mesophase.

\section{Conclusions}

ATR-FTIR spectroscopy is shown to be an efficient technique to investigate the dynamics of surfactant aggregation and surfactant-silicate mesophase formation. This method allowed determining a CMT value for a $2.1 \mathrm{wt} \%$ PE10400 in nitric acid media close to that obtained at neutral $\mathrm{pH}$. The silicate formation, precipitation of silicatesurfactant flocs and further silicate condensation have been monitored during the synthesis of SBA-15.

\section{Acknowledgment}

MTS gratefully acknowledges the Spanish Ministry of Education and Science (MEC) for a PhD grant. This work has been supported by MEC (project CTQ2006-06282).

\section{References}

[1] J. Patarin, B. Lebeau, R. Zana, Curr. Opin. Colloid Interf. Sci., 7 (2002) 10

[2] Y. Wan, D. Zhao, Chem. Rev., 107 (2007) 2821

[3] M.T. Sánchez, J. Pérez-Pariente, C. Márquez-Álvarez, Stud. Surf. Sci. Catal., 158 (2005) 113

[4] Y.L. Su, J. Wang, H.Z. Liu, J. Colloid Interf. Sci., 251 (2002) 417

[5] Y.L. Su, J. Wang, H.Z. Liu, J. Phys. Chem. B, 106 (2002) 11823

[6] M. Milosevic, Appl. Spectr. Rev., 39 (2004) 365

[7] P. Alexandridis, J.F. Holzwarth, T.A. Hatton, Macromolecules, 27 (1994) 2414

[8] K. Flodström, H.Wennerström, V. Alfredsson, Langmuir, 20 (2004) 680 